\title{
HABILIDADES PROFISSIONAIS COLABORATIVAS PARA ENGENHEIROS POR MEIO DA GEOMETRIA DESCRITIVA
}

\author{
COLLABORATIVE PROFESSIONAL SKILLS FOR \\ ENGINEERS THROUGH DESCRIPTIVE \\ GEOMETRY
}

\author{
Ana Cláudia Rocha \\ Cavalcanti \\ Dra. em Desenvolvimento \\ Urbano \\ Universidade Federal de \\ Pernambuco, \\ Departamento de \\ Expressão Gráfica, Recife, \\ Brasil \\ ana.rcavacanti@ufpe.br
}

Vandré Ricardo Pereira

Melo

Licenciado em Expressão

Gráfica

vandrecgi@yahoo.com.br

Flávio Antonio Miranda de Souza

$\mathrm{PhD}$ em Planejamento

Urbano

Universidade Federal de

Pernambuco,

Departamento de

Expressão Gráfica, Recife,

Brasil

flavio.desouza@ufpe.br

\begin{abstract}
RESUMO:
Os desafios profissionais contemporâneos nos campos das engenharias têm demandado novas habilidades em lidar com a forma, tanto nos meios de representação tradicionais e como nos meios digitais, dos quais, o lápis e papel e os aplicativos de auxílio ao projeto são coexistentes e complementares. A modelagem de objetos tem que ser precisa, antecipando erros para evitar desperdícios. A correção antecipada desses erros em sua fase projetual é, sem dúvida, fundamentada no conhecimento da geometria descritiva. Do ponto de vista das habilidades interpessoais, novas demandas têm sido freqüentes, tais como cooperação, colaboração, interatividade, entre outras. Portanto, esse artigo discute as experiências do ensino da geometria descritiva nos cursos de engenharia civil e engenharia mecânica da Universidade Federal de Pernambuco. 0 estudo faz uso de metodologias ativas de ensino e aprendizagem para a aquisição de visualização espacial que estimulam a colaboração para aprender a aprender num cenário de constantes inovações. Os estudantes utilizaram ferramentas tradicionais de desenho (lápis e papel) e ferramentas digitais (AutoCAD e SketchUp) aplicadas na resolução de problemas. A inovação refere-se ao uso de metodologias ativas para a apreensão de conteúdos e habilidades colaborativas. Os estudos apresentados demonstram que os estudantes assumiram papeis ativos na aquisição do conhecimento, interagindo e colaborando com diferentes grupos de trabalho.
\end{abstract}

Palavras-chave: Metodologias ativas; geometria descritiva; engenharias.

\section{ABSTRACT:}

The contemporary challenges of professionals in the fields of engineering have demanded new skills in dealing with form, both in the traditional means of representation and in the digital media, of which the pencil and paper and tools of Computer Aided Design coexist and are complementary. Object modeling has to be accurate, anticipating errors in order to avoid reworking. The correction of errors in the design phase is undoubtedly grounded in the knowledge of descriptive geometry. From the point of view of interpersonal skills, new demands frequently have been present such as cooperation, collaboration, interactivity, among others. This article discusses teaching experiences of descriptive geometry in the courses of civil engineering and mechanical engineering at Federal University of Pernambuco. The study makes use of active methodologies through innovative tools to broaden the debate about the applications of descriptive geometry in the design of models and the active teaching and learning methodologies for the acquisition of spatial visualization that stimulate collaboration in the learning process in a scenario of constant innovation. The students used traditional drawing tools (pencil and paper) and digital tools (AutoCAD and SketchUp) applied in solving problems. The innovation refers to the use of active methodologies for the apprehension of contents and the development of collaborative skills. These studies demonstrate that students have interacted and collaborated actively with different working groups.

Keywords: Active methodologies; descriptive geometry; engineering. 


\section{INTRODUÇÃo}

De modo geral e em nível global, as empresas têm procurado por profissionais que possuam a habilidade de trabalhar efetivamente em equipe, se adaptando à cultura do ambiente de trabalho e, que consigam transmitir ideias de forma eficiente (EUROPEAN COMMISSION, 2013). Entretanto, de modo quase contraditório, os currículos tradicionais nas universidades não estão focando de forma substancial o desenvolvimento dessas competências. Apesar disso, diferentes experiências de ensino vêm ocorrendo em adaptação às recentes inovações, tentando responder às crescentes demandas profissionais advindas em grande parte das transformações de produção de bens e de consumo, assim como das formas de organização da sociedade, dos meios de comunicação digital, das formas de ensinar e aprender, entre outras.

Um número crescente de experiências didático-pedagógicas pode ser observado por meio de estratégias que utilizam os princípios da Aprendizagem Baseada em Projetos (PBL) no ensino universitário em diferentes países (CHANDRASEKARAN et al, 2012), incluindo o Brasil (GIANNOTTI et al, 2008). O PBL lida com o enfretamento direto à aprendizagem baseada em problemas (RIBEIRO, 2008). Também pode ser considerado um ambiente de aprendizado onde o problema motiva os alunos a aprender. PBL consiste numa abordagem de ensino colaborativa, construtivista e contextualizada que utiliza problemas na vida real para motivar a construção do conhecimento (BARROWS, 1996), influenciada por fatores contextualizados que dependem da interação entre os alunos que interagem de forma ativa na busca de resoluções dos projetos.

A abordagem do PBL é delineada nos princípios derivados da psicologia cognitiva, caracterizada como uma forma de aprendizagem e instrução colaborativa, construtivista e contextual (MAMEDE, 2001), estando relacionada com a teoria construtivista da aprendizagem (LEVIN, 2001).

Por sua vez, o construtivismo, no campo da educação, ressalta que o indivíduo transforma as informações do mundo em sua mente por meio da manipulação das informações que lhes são apresentadas, com os propósitos de que cada estudante possa construir seu conhecimento. Para tanto, diversos experimentos têm sido feitos no sentido de mostrar como ocorrem os desenvolvimentos cognitivos dos indivíduos, ressaltando a participação do sujeito na formação do conhecimento (CARVALHO et al, 2017). Portanto, a relação entre o objeto real e a sua representação apresenta características de esquemas de transformações produzidas pelos próprios estudantes, que rompem com a relação da apreensão dos conhecimentos na relação do ensino e da aprendizagem, sendo necessário, portanto, que os professores desenvolvam estratégias que possibilitem facilitar a articulação e o acionamento dos esquemas de enfrentamento dos problemas elaborados pelos estudantes. 
No processo de ensino e aprendizagem há uma prática social complexa entre alunos e professores que engloba tanto a ação de ensinar como de aprender (PIMENTA e ANASTASIOU, 2002). Tal processo não se resume ao momento de sala de aula (LIBÂNEO, 1990), ou seja, inclui o planejamento, as ações participativas dos sujeitos (alunos e professores) propriamente ditas em sala, os acompanhamentos e as avaliações.

Diversas enquetes foram realizadas ao longo dos semestres entre 2016 e 2018 com o propósito de diagnosticar a situação dos estudantes quanto aos processos de ensino e aprendizagem. Logo no primeiro dia de aula, aplica-se um teste de visualização espacial baseado em Montenegro (2003). Observa-se que os alunos apresentam um bom nível de visualização de sólidos geométricos. Busca-se acompanhar as dinâmicas dos processos de ensino-aprendizagem, envolvendo atividades complexas, construção de significados e interações entre concepções, conceitos e contextos de aprendizagem (MARTÍNES, 2008). A participação do estudante como agente proativo no processo de ensino-aprendizagem se faz cada vez mais necessária, principalmente diante do cenário de novas tecnologias e ferramentas que nos permitem acessar cada vez mais informações de maneira rápida e eficiente. Diante disso, exige-se, mas nem sempre com êxito, uma maior participação dos estudantes em sala de aula, interagindo e trocando experiências que, por sua vez, influência e repercute diretamente no processo de ensino e aprendizagem (CAVALCANTI et al, 2015; 2016).

Estratégias inovadoras quanto ao uso de recursos didáticos tem ampliado os canais de diálogos entre professores e alunos, tais como, uso de ferramentas computacionais gráficas, disponibilização prévia de literatura específica para os alunos, além de ambientes de ensino colaborativos, entre outras coisas, tem estimulado professores e estudantes a saírem de suas zonas de conforto, tornando os ambientes de sala de aula mais realistas quanto aos desafios a serem enfrentados em suas vidas fora da sala de aula, proporcionando situações didáticas frente a sujeitos mais inquietos e pensantes Dessa forma, foi selecionado como recurso didático a utilização e produção de modelos físicos e digitais para o ensino de geometria descritiva aplicados à engenharia civil e engenharia mecânica, levando em consideração os diferentes perfis de cada curso, dos estudantes e as habilidades demandadas por cada curso (CAVALCANTI et al, 2018).

Por meio de modelos didáticos busca-se reproduzir a realidade de forma esquematizada e concreta, facilitando a compreensão do estudante, uma vez que possibilita a visualização e manipulação de formas geométricas, ao mesmo tempo em que aumenta o interesse dos estudantes para a experimentação de conteúdos relacionando teoria e prática. 0 uso de modelos proporciona uma maior apreensão para a aprendizagem já que atuam como método ativo de aprendizagem capaz de envolver todos os participantes ao mesmo tempo na mesma tarefa. Os modelos ainda podem ser baratos e de execução relativamente rápida, possibilitando que cada 
aluno produza seu próprio modelo, desenvolva habilidades, competências e atitudes (CAVALCANTI e SILVA, 2008).

Em relação ao uso de elementos visuais, Prendes Espinosa (1996) conclui que eles têm um enorme potencial para transmitir diferentes conceitos, muitas vezes de forma mais clara do que por meio do uso da linguagem natural, ou seja, por meio de palavras.

Portanto, este trabalho investiga como e em que circunstâncias, o uso de elementos visuais físicos (modelos tridimensionais físicos e digitais) possibilita a aprendizagem ativa por parte dos estudantes por meio da experimentação dos conteúdos de forma a refletir sobre a teoria e a prática da aplicação da geometria descritiva em objetos manipuláveis, como na construção de sólidos geométricos, telhados e superfícies topográficas. Espera-se determinar como e por que os modelos facilitam a adequação de significados ao processo de aprendizagem.

\section{GEOMETRIA DESCRITIVA E NOVAS FERRAMENTAS.}

A Geometria Descritiva é um método de estudo de objetos em três dimensões representados no plano que é bidimensional, desafiando a capacidade intelectual dos estudantes na visualização espacial. Portanto, um aspecto fundamental nesse trabalho consiste na investigação de alternativas didáticas que visem ampliar a capacidade de visualização espacial por parte dos alunos de engenharia mecânica e civil, por meio de conteúdos conceituais e procedimentais trabalhados na disciplina de geometria descritiva.

A capacidade de visualização espacial e as habilidades de rotação mental de objetos são essencialmente importantes para profissionais que se dedicam às engenharias (STACHEL, 2005; SEABRA e SANTOS, 2007; CAVALCANTI et al, 2016). E, por meio do desenvolvimento de habilidades de visualização espacial os profissionais de engenharias podem interagir melhor e aproveitar interfaces computacionais que influenciam na capacidade de aprender de forma eficaz a usar programas do tipo Computer Aided Design (CAD).

A visualização espacial envolve a habilidade de imaginar e, mentalmente transformar informações espaciais (UTALL et al, 2013; SEABRA e SANTOS, 2007), sendo influenciada por treinamento. Esse estudo adota uma visão exploratória para ampliar capacidades e habilidades por meio de estratégias que estimulem o envolvimento dos alunos de forma inovativa, participante e colaborativa como o PBL. O PBL foi utilizado na tentativa de favorecer um ambiente onde as interações entre os alunos ajudem a estimular e aguçar as habilidades de visualização espacial dos alunos de engenharia civil e de engenharia mecânica.

A amostra desse estudo consiste de três turmas de Geometria Descritiva, sendo duas do curso de engenharia civil e uma do curso de engenharia mecânica, durante o segundo semestre de 2017. 0 número total 
de alunos matriculados nas turmas é de 80 alunos. A metodologia adotada foi de observação participante em sala de aula, comparando meios tradicionais de ensino da geometria descritiva e projetos de resolução de problemas de geometria descritiva.

Em estudo recente sobre novas experiências de trabalhos que demandam ações colaborativas na aplicação de conteúdos programáticos da geometria descritiva, Cavalcanti et al (2015) concluem indicando que houve um crescente envolvimento nas atividades de resolução de problemas por parte dos estudantes nas atividades de classe, e ainda demandando atitudes colaborativas, mesmo nas atividades didáticas utilizando técnicas tradicionais de fabricação de modelos físicos assim como na modelagem digital dos modelos.

Além disso, diante de desafios que demandaram ações mais ativas de participação para a resolução de problemas, os alunos necessitaram buscar mais informações sobre os projetos nos quais estavam envolvidos e se organizarem individualmente, como também necessitaram coordenar, em grupo, as atividades de cada um deles, as atividades realizadas em pares, em grupos, etc. (CAVALCANTI et al, 2016). Para tanto, os estudantes tomaram consciência do que estavam descobrindo ao realizarem as atividades, e principalmente, tornaram-se protagonistas de suas escolhas e decisões ao resolverem os problemas. A aplicação dos conteúdos programáticos da geometria descritiva em problemas resolvidos por meio de construção/manipulação de sólidos geométricos e modelos auxiliaram positivamente na apreensão desses conteúdos. Esse trabalho dá continuidade a tais experimentos, aprofundando questões sobre alternativas de como estimular os alunos para a apreensão dos conteúdos da geometria descritiva e melhorar suas habilidades espaciais visuais, uma vez que tais habilidades melhoram significativamente na aprendizagem nos campos das ciências, tecnologias, engenharia e matemática (UTTAL et al, 2013).

0 ensino de geometria descritiva nas engenharias tem passado por um processo de adaptação voltado a acompanhar os desafios profissionais contemporâneos, ao mesmo tempo em que novas ferramentas computacionais facilitam a interação dos usuários a programas e periféricos que auxiliam na fabricação e manipulação de sólidos e modelos (digitais e físicos), tais como cortadoras lasers, impressoras 3D, etc. Além disso, cada vez mais se demanda dos profissionais nas diferentes áreas atitudes proativas, colaborativas e flexíveis.

\section{O PBL E A GEOMETRIA DESCRITIVA}

Neste trabalho destacamos o papel de abordagens teóricometodológicas conhecidas por ferramentas ligadas ao PBL, amplamente utilizada na escola construtivista que pode também ser empregada no ensino superior universitário como forma de apontar novos rumos para o ensino da geometria descritiva.Num estudo sistemático da aplicação de ferramentas de 
aprendizagem baseadas em PBL para o ensino de projetos para alunos de engenharia civil na Irlanda, Gavin (2011) concluiu que os alunos puderam vivenciar experiências que estimularam habilidades para: resolução de problemas, proposição com inovação, desenvolver trabalhos em grupos e apresentação oral de suas propostas. Segundo de Bono (1992), o ambiente colaborativo demandando soluções criativas tem sido resultado do aumento do ambiente competitivo promovido pela globalização da economia e pela internacionalização do ambiente corporativo.

Assim sendo, estratégias pedagógicas que desenvolvam essas habilidades durante o ensino da graduação podem auxiliar na inserção do mercado de trabalho cada vez mais competitivo. As qualidades de adaptabilidade e flexibilidade em enfrentar problemas do dia a dia têm sido valoradas cada vez mais no ambiente corporativo.

Daí, a preocupação em propor situações de trabalhos colaborativos e inovativos que estimulem os alunos a aprenderem a aprender, como estratégia de enfretamento dos problemas têm sido tão valoradas nestas experiências pedagógicas. Atribui-se à experiência do PBL como ferramenta inovadora no ensino de graduação no curso de medicina no Canadá como sendo o início dessas experiências no ensino superior (BARROWS, 2000).

Uma das questões primordiais quanto ao uso de PBL seria o papel ativo por parte dos estudantes na construção do conhecimento em grupos colaborativos (HMELO-SILVER, 2004), alem de ser fundamental na mudança de papel entre os docentes e entre os docentes e estudantes.Inúmeras experiências nos diversos setores têm sido experimentadas, como o caso do ensino da engenharia na Irlanda (GAVIN, 2011). No Brasil há outras experiências do uso de PBL nas escolas de engenharia, alem de em outros cursos, relatadas por SEABRA; SANTOS (2007), CAVALCANTI et al (2016), entre outros. Esse estudo amplia o debate e indica novas alternativas ao processo de ensino-aprendizagem da geometria descritiva.

\subsection{Aplicando geometria descritiva na engenharia mecânica}

Nos trabalhos desenvolvidos com a turma de engenharia mecânica, (Figuras 1, 2 e 3), as atividades foram desenvolvidas em grupos de 3 a 4 estudantes, atendendo aos princípios colaborativos aplicados para a resolução de problemas. Os projetos foram desenhados para aplicar os conhecimentos de geometria descritiva e, ao mesmo tempo, proporcionar ambientes de discussão e de resolução de problemas.

Figura 1 - Resolução de problemas de forma colaborativa com estudantes de engenharia mecânica.

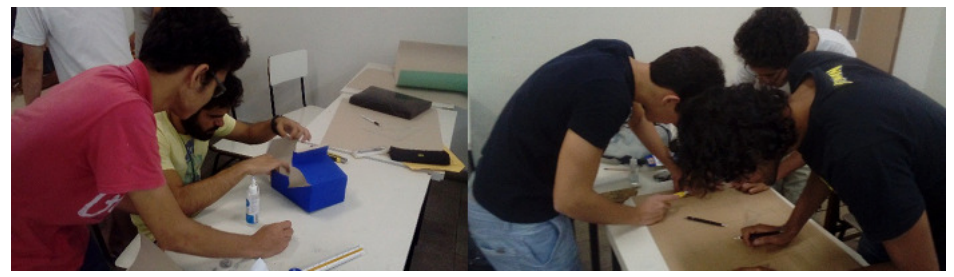

Fonte: Os autores. 
Nesses trabalhos os estudantes são desafiados a compreenderem antecipadamente formas tridimensionais a partir de conhecimento básico da geometria tridimensional e devido à exposição de exercícios de resolução de problemas de forma tradicional. Aos poucos, os estudantes se deparam com novas situações problemas que demandam um aprofundamento do conhecimento aplicado da geometria descritiva, como por exemplo, interseção de planos, determinação de verdadeira grandeza de seção plana, entre outras situações problemas (Figuras 1 e 2).

Figura 2 - Aplicando os conteúdos em problemas para estudantes de engenharia mecânica.

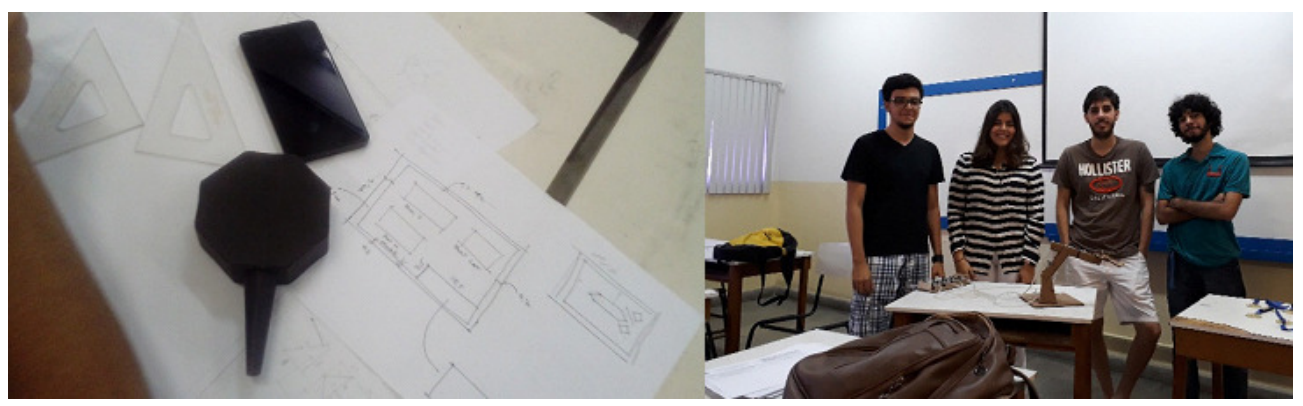

Fonte: Os autores.

Na Figura 3, os problemas foram incrementados por meio de sólidos mais complexos e que se assemelham a peças mecânicas para atenderem a demandas específicas de engenharia mecânica, demandando mais atenção e participação dos estudantes para a resolução dos mesmos. As situações problemas exigiram dos estudantes aplicarem os conteúdos de forma a estimular a criatividade e estabelecer estratégias para resoluções de problemas aplicadas aos sólidos complexos, como modelos reduzidos de automóveis, etc.

Nessas experiências de ensino-aprendizagem da geometria descritiva foram consideradas as inovações que ocorrem e perpassam os conteúdos de aprendizagem conceituais, procedimentais e atitudinais.

Figura 3 - Aplicação de resolução de problemas e modelos tridimensionais.

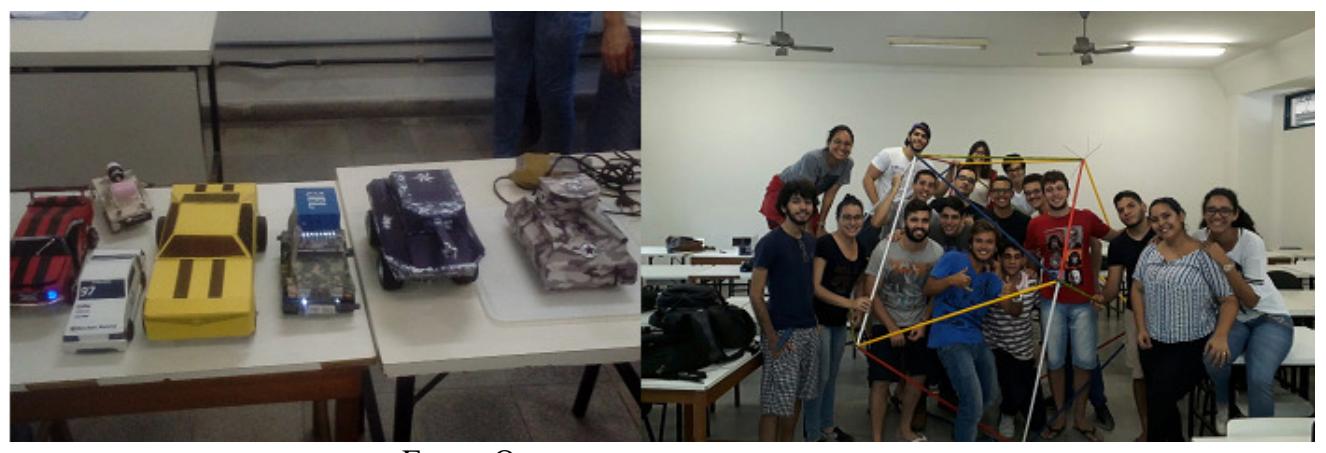

Fonte: Os autores.

Essas experiências utilizando metodologias ativas para o desenvolvimento de habilidades para a visualização espacial demonstram ser bastante significativas ao auxiliarem os alunos a aprender a aprender, num cenário de constantes transformações. 


\subsection{Geometria descritiva aplicada na engenharia civil}

Em consonância com o estudo realizado com a turma de engenharia mecânica, os projetos realizados com os alunos de engenharia civil foram voltados para aplicar os conhecimentos da geometria descritiva nas suas áreas de atuação. Os estudantes foram demandados a resolverem os problemas de forma colaborativa.

A Figura 4 mostra o resultado diversificado na solução apresentada para soluções de problemas com interseção de planos. A aplicação dos conteúdos demandou principalmente colaboração entre os membros dos grupos, definição de estratégias de resolução de problemas e criatividade por meio da exploração de materiais, cores, texturas, volumes. Elementos que não são medidos apenas com o produto, mas por meio do acompanhamento sucessivo dos processos.

Figura 4- Resolução de problemas aplicados à engenharia civil.

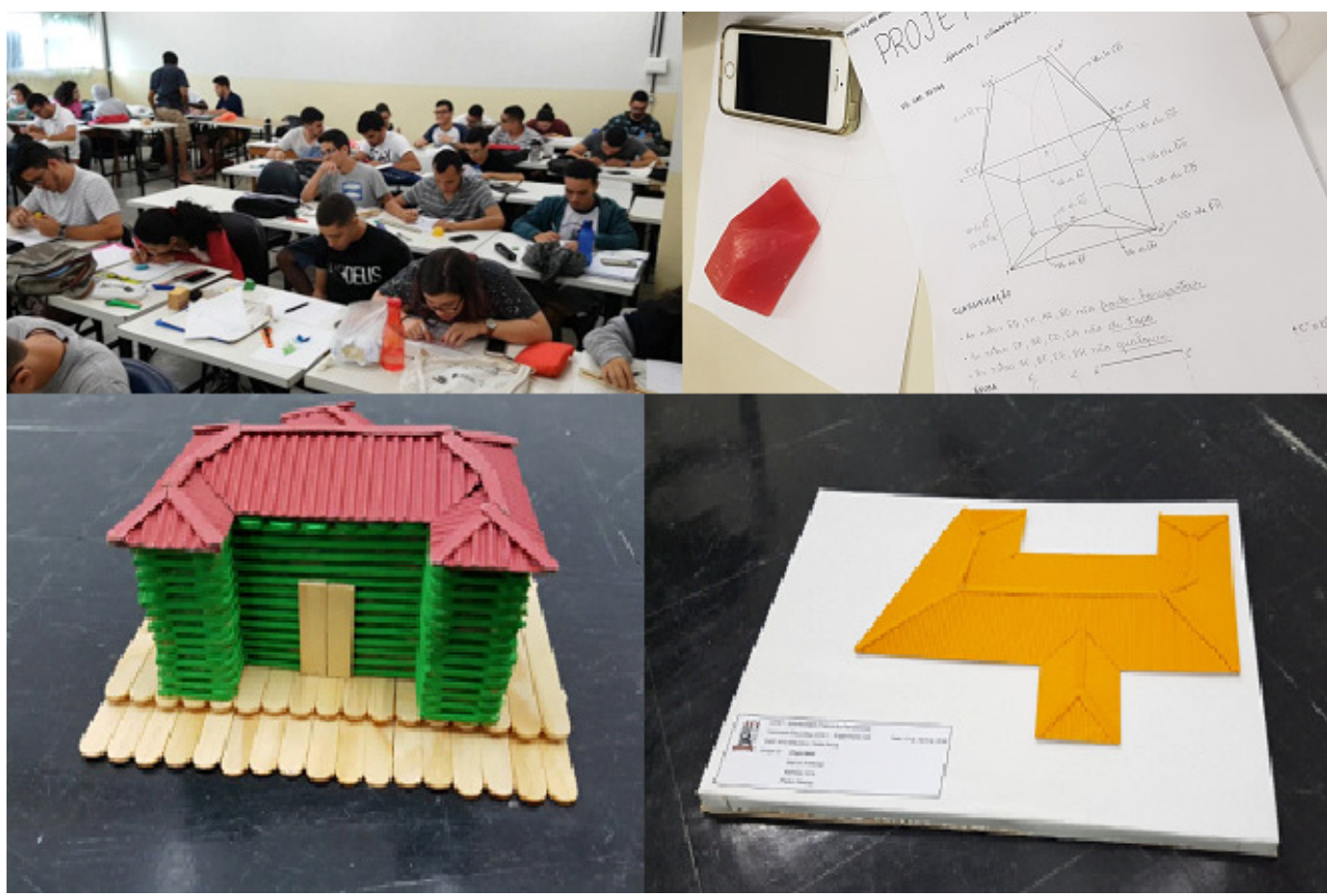

Fonte: Os autores.

A interação entre as mídias tradicionais e digitais (Figura 5) contribuiu para o desenvolvimento de habilidades de visualização espacial na medida em que os estudantes comparavam e contrastavam os modelos nas duas mídias. Ao mesmo tempo, as atividades desenvolvidas em equipes demandaram dos seus membros a divisão de atividades e, em alguns casos, ocorrendo em erros na resolução de problemas. Ao errarem foi possível corrigir no meio digital de forma mais rápida do que no meu tradicional e, com isso os alunos aprenderam com o erro, seguido de novas alternativas. 
Figura 5- Produção de modelos tridimensionais digitais e físicos com estudantes de engenharia civil.

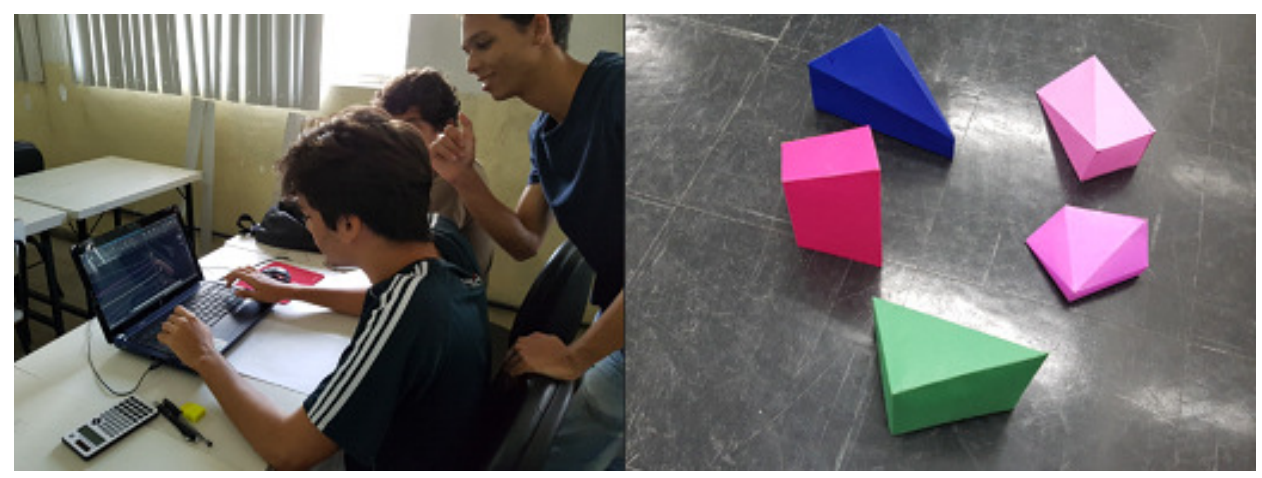

Fonte: Os autores.

No caso de trabalhos com modelos físicos para a resolução de superfícies topográficas (Figura 6), uma grande gleba foi dividida em 14 partes colindantes. Cada parte da superfície total se interligava com outras duas ou três áreas vizinhas. Portanto, as equipes tiveram que trabalhar em conjunto para definir estratégias colaborativas, visando a determinação de um greide para uma estrada de acesso principal que cortava toda a superfície. Nessas atividades inúmeras vezes, diferentes estudantes atuaram de forma a liderar a solução dos problemas.

Figura 6- Aplicação dos conteúdos para estudantes de engenharia civil: Situação problema, colaboratividade e liderança.

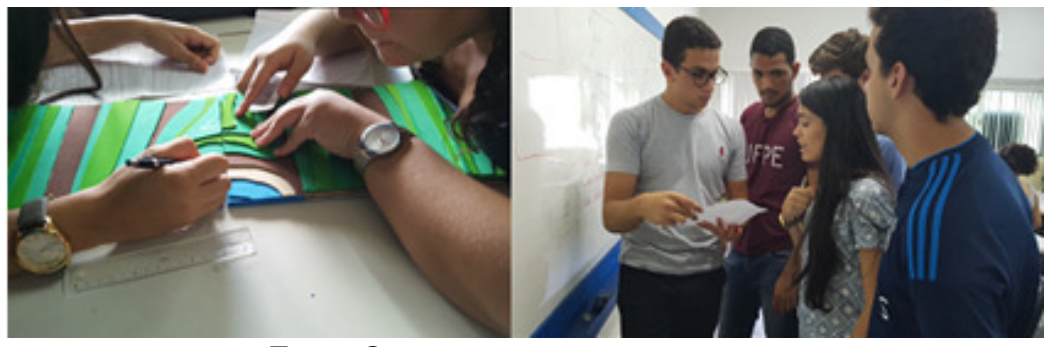

Fonte: Os autores.

Observa-se que durante as atividades em grupo há necessidade de coordenação e distribuição interna de atividades para aperfeiçoar os processos de realização dos projetos que envolvem muitos procedimentos e que podem comprometer o aprendizado.

Do ponto de vista da compreensão dos conteúdos programáticos de geometria descritiva e da utilização e domínio de ferramentas digitais em trabalhos em grupo, aponta-se para o engajamento necessário de todos os membros das equipes visando minimizar as dificuldades individuais em lidar com as ferramentas digitais e manipulação de modelos físicos (Figura 7). Para tanto, é de extrema importância o domínio dos conteúdos de geometria descritiva individualmente, que poderá colaborar na resolução digital dos problemas.

Ao observarmos o caso em estudo, se por um lado os estudantes se apropriaram de forma autônoma das estratégias de resolução de problemas 
diante das incertezas, por outro lado, ao lidar com incertezas na realização de uma atividade que tem caráter avaliativo, os estudantes se sentiram inseguros, e por vezes, desmotivados e resistentes. Entretanto, observações em sala de aula nos levam a perceber que os entraves foram gradativamente superados por meio de contínua tentativa de resolver os projetos.

Para os alunos, há necessidade de coordenação e distribuição interna de atividades para aperfeiçoar os processos de realização dos projetos que envolvem muitos procedimentos e que podem comprometer o aprendizado.

Figura 7- Colaboração, coordenação de atividades para solução de problemas.

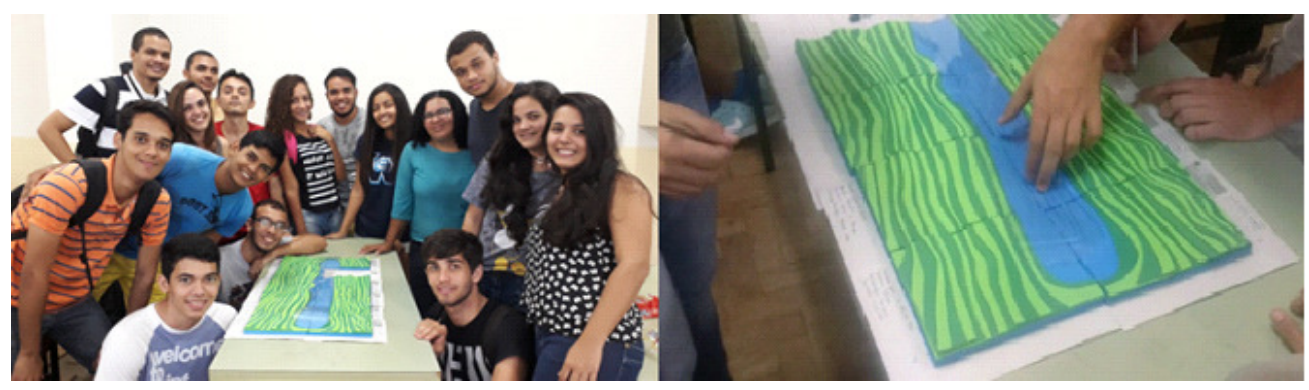

Fonte: Os autores.

Nem sempre os alunos apresentam iniciativa própria de forma persistente. Improvisações motivacionais se fazem necessárias durante as interações entre alunos e professores, tais como mostrar exemplos de trabalhos de profissionais que resolveram, de forma criativa e inovativa, exemplos de projetos semelhantes ao trabalhado em sala, quando possível. Ou até mesmo mostrar e comentar os trabalhos dos colegas e questionar sobre os problemas enfrentados nesses trabalhos.

\section{CONCLUSÕES}

A experiência aqui relatada fez uso de ferramentas tradicionais de trabalho e de representação gráfica além de ferramentas digitais. As ferramentas digitais estimularam os alunos a trabalharem de forma colaborativa e serviram para auxiliar na visualização espacial. Além disso, o uso de ferramentas computacionais demonstrou ser excelente para auxiliar no aumento do potencial de visualização espacial, porém, não devem ser substitutivas das capacidades mentais utilizadas para resolução de problemas que demandam raciocínio espacial por meio da geometria gráfica. 0 uso de ferramentas computacionais pode, entretanto, auxiliar na otimização do potencial exploratório de resolução de problemas gráficos, principalmente nas etapas de aprimoramento de modelos tridimensionais.

Entretanto, a inovação abordada na disciplina foi relativa ao uso de metodologias colaborativas (PBL). 
Mesmo quando os estudantes apresentaram altos índices de sucesso na resolução de problemas gráficos, as abstrações exigidas para o uso de resolução de problemas por meio da geometria clássica serviu para desafiar os conhecimentos e a antecipação de resultados em resolução gráfica por meio do uso de ferramentas tradicionais, além do esperado, atuando como estratégia de desenvolver a visão espacial dos estudantes de forma bastante satisfatória.

A produção de modelos físicos auxiliou na visualização de conteúdos abstratos por meio da constante comparação entre as noções de ponto, reta e plano com os objetos concretos, assim como, por meio da manipulação de sólidos preexistentes, os conteúdos foram adquiridos. As relações interpessoais parecem ter sido estreitadas e atitudes de liderança foram revezadas entre os componentes das equipes. Por vezes, as atividades foram realizadas individualizadas, mas coordenadas. Por vezes, as discussões ocorriam entre os membros das equipes, e até mesmo entre equipes, pela curiosidade em ver os outros trabalhos.

0 processo de conscientização da aprendizagem não é linear, assim como o da própria aprendizagem, nem é linear a tomada do protagonismo. Enfatizamos ser primordial a consciência de que os alunos aprendem fazendo, sendo fundamental aprender a identificar os diferentes conteúdos adquiridos ao longo do processo de aprendizado e a tomada de consciência das descobertas ao realizarem as atividades, e principalmente, a consciência que os estudantes tornam-se protagonistas de suas escolhas e decisões ao resolverem os problemas.

Os erros ocorridos ao longo do processo de resolução de problemas são fundamentais para a aprendizagem dos conteúdos programáticos e de novas atitudes perante situações de frustração. Os estudantes deixam de ser receptáculos passivos do conhecimento transferido pelo ensino tradicional. Cada vez mais as dúvidas eram compartilhadas e o aprendizado era adquirido de forma colaborativa. Ao mesmo tempo, os professores assumiram posições proativas no processo e colaboram com os estudantes ao estimularem novas formas de aquisição do conhecimento.

Por fim, esse trabalho conclui que as metodologias ativas aqui utilizadas contribuirão para servir como base para as atuações profissionais dos futuros engenheiros. Conclui ainda que os docentes serviram como facilitadores do processo de resolução de problemas, interagindo de forma mais proativa no contexto de metodologias ativas.

\section{REFERÊNCIAS}

BARROWS, H. S. Problem-based learning in medicine and beyond: A brief overview. In: WILKERSON, L.; GIJSELAERS, W. H. (Eds.). Bringing problembased learning to higher education: theory and practice. San Francisco: Jossey-Bass, 1996, p. 3-12. 
BARROWS, H. S. Problem-based learning applied to medical education. Springfield, IL: Southern Illinois University Press, 2000.

BONO, E. Serious creativity: using the power of lateral thinking to create new ideas. London, Harper Collins. 1992.

CARVALHO, GISELE L. de et al. The Integration of Graphic Disciplines in Civil Engineering Courses through Computer Graphics. Journal of Mechanics Engineering and Automation. v. 7, p. 94-100, 2017.

CAVAlCANTI, A. C. R.; De SOUZA, F. A. M.; CARVALHO, G. L. Processos colaborativos na aprendizagem da geometria descritiva para o curso de engenharia civil. In: XLIII CONGRESSO BRASILEIRO DE EDUCAÇÃO EM ENGENHARIA, 2015, Mauá. Anais... Mauá-RJ, 2015.

CAVALCANTI, A. C. R. et al. Habilidade espacial e colaborativa dos estudantes de engenharia civil: adaptando PBL para o ensino-aprendizagem de geometria descritiva. In: CONGRESSO BRASILEIRO DE EDUCAÇÃO EM ENGENHARIA, 44., 2016, Natal. Anais... Natal-RN, 2016.

CAVAlCANTI, A. C. R.; MELO, V. R. P.; De SOUZA, F. A. M. Contribuição da geometria descritiva para uma educação inovadora nas engenharias mecânica e civil. In: XLVI CONGRESSO BRASILEIRO DE EDUCAÇÃO EM ENGENHARIA, 46., 2018, Salvador. Anais... Salvador, 2018.

CAVALCANTI, Danuza; SILVA, Aparecida. Modelos didáticos de professores: concepções de ensino-aprendizagem e experimentação. In: XIV ENCONTRO NACIONAL DE ENSINO DE QUÍMICA, 2008, Curitiba. Anais... Curitiba, 2008.

CHANDRASEKARAN, S. et al. Learning through projects in engineering education. In: SOCIETÉ EUROPÉENNE POUR LA FORMATION DES INGÉNIEURS ANNUAL CONFERENCE, 40., 2012, Thessaloniki. Anais... Thessaloniki: SEFI, 2012.

EUROPEAN COMMISSION. European classification of skills/competences, qualifications and occupations. Publication Office of the European Union, Luxembourg, 2013.

GAVIN, K. Case study of a project-based learning course in civil. European Journal of Engineering Education, No. 36, Vol. 6, p. 547-558. 2011.

GIANNOTTI, M. et al. Proposta de aplicação do PBL nos cursos de engenharia. In: CONGRESSO BRASILEIRO DE EDUCAÇÃO EM ENGENHARIA, 36., 2008, São Paulo. Anais... São Paulo: ABENGE, 2008.

HMELO-SILVER, Cindy E. Problem-Based Learning: what and how do students learn? Educational Psychology Review, v.16, n.3, 2004, p. 235266.

LEVIN, B. Energizing teacher education and professional development with problem-based learning. United States: ASCD, 2001.

LIBÂNEO, J. C. Didática. São Paulo: Cortez, 1990. 
MAMEDE, S. Aprendizagem baseada em problemas: características, processos e racionalidade. In: MAMEDE, S.; PENAFORTE, J. (Orgs.). Aprendizagem baseada em problemas: anatomia de uma nova abordagem educacional. Fortaleza: Hucitec, 2001. p. 25-48.

MATÍNEZ, A. M. A criatividade como princípio funcional da aula: limites e possibilidades. In: VEIGA, I. P.A. (Org.) Aula: gênese, dimensões, princípios e práticas. Campinas: Papicus, 2008. p. 115-143.

MONTENEGRO, G. Habilidades espaciais: exercícios para o despertar de ideias. Santa Maria: sCHDs, 2003.

NORMAN, K. L. Spatial visualization: a gateway to computer-based technology. Journal of Special Educational Technology, v. XII, n. 3, 1994, p. 195-206.

PIMENTA, S. G.; ANASTASIOU, L. D. G. C. Docência no ensino superior. São Paulo: Cortez, 2002.

PRENDES ESPINOSA, Maria Paz. Análisis de imágenes en textos escolares. Pixel-Bit. Revista de Medios y Educación, 1996, n. 6, p. 15-39.

SEABRA, Rodrigo; SANTOS, Eduardo. Avaliando a aptidão espacial de estudantes em um curso de geometria gráfica. In: VII INTERNATIONAL CONFERENCE ON GRAPHICS ENGINEERING FOR ARTS AND DESIGN, 2007, Curitiba. Anais... Curitiba, 2007.

STACHEL, Hellmuth., Descriptive Geometry in today's engineering curriculum. Transactions of FAMENA Vol. 29 N. 2 , 2005, pp. 35-44.

UTTAL, D. et al. The malleability of spatial skills: a meta-analysis of training studies. Psychological Bulletin, 2013, v. 139, n. 2, p. 352-402.

\section{AGRADECIMENTOS}

Somos muito gratos aos estudantes pela confiança, colaboração e cumplicidade nas experiências compartilhadas nas salas de aula. 